\title{
Nuclear Information Services at the National Nuclear Data Center
}

\author{
Thomas W. Burrows and Charles L. Dunford \\ National Nuclear Data Center, Brookhaven National Laboratory, Upton, NY 11973-5000, USA
}

\begin{abstract}
The National Nuclear Data Center has provided remote access to its databases and other resources since 1986. This year we have completed the modernization of our databases and Web site. Resources available from our Web site will be summarized and some of the major improvements described in more detail.
\end{abstract}

\section{INTRODUCTION}

The National Nuclear Data Center (NNDC) has been providing remote access to the nuclear physics databases it maintains and to other resources since 1986. Access was originally via DECNET or modem; then by TELNET, the successor to DECNET; and more recently the Web and FTP; and now exclusively through the Web.

Since about 1999, the NNDC and a collaborator from the IAEA Nuclear Data Section have been working to migrate the databases from the original CODASYL-DBMS to a modern relational database system. The Web interfaces to these databases have been upgraded to use Simple Query Language (SQL)

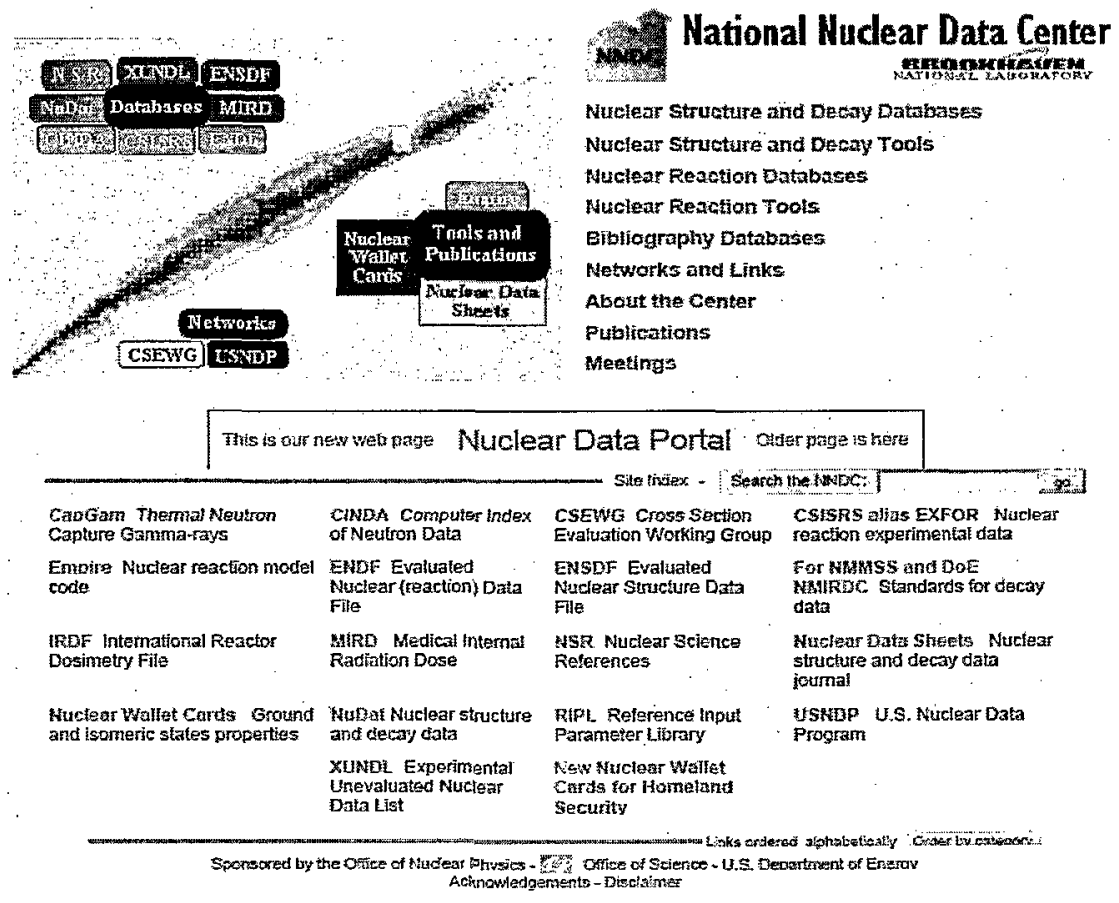

FIGURE 1. New National Nuclear Data Center Home Page, www.nndc.bnl.gov 


\begin{tabular}{|c|c|c|}
\hline Database & URL & Description \\
\hline CINDA & www.nndc.bnl.gov/cinda/ & $\begin{array}{l}\text { Computer Index to Nuclear Data: Bibliographical neutron induced reaction } \\
\text { information, including experimental, theoretical, and evaluation works. It contains } \\
\text { references to } 275,000 \text { reactions from } 55,000 \text { works. }\end{array}$ \\
\hline CSISRS & www.nndc.bnl.gov/exfor/ & $\begin{array}{l}\text { Cross Section Information Storage and Retrieval System }{ }^{2} \text { Experimental } \\
\text { nuclear reaction data for incident neutrons, charged particles, and photons. It } \\
\text { covers more than } 14,000 \text { experiments and is considered "complete" for neutron- } \\
\text { induced reaction data. }\end{array}$ \\
\hline ENDF & www.nndc.bnl.gov/endf/ & $\begin{array}{l}\text { Evaluated Nuclear Data File: }{ }^{3} \text { Recommended reaction data from the ENDF/B- } \\
\text { VI, JEFF, JENDL, BROND, and CENDL libraries. It provides data in the ENDF-6 } \\
\text { format, covering all nuclides of practical relevance for neutrons up to } 20 \mathrm{MeV} \text { and } \\
\text { partly up to } 150 \mathrm{MeV} \text {. It serves as principal input for neutronics calculations, } \\
\text { including nuclear reactor design, national security, accelerators, criticality safety, } \\
\text { shielding, radiation protection, and detector simulation. }\end{array}$ \\
\hline ENSDF & www.nndc.bnl.gov/ensdf/ & $\begin{array}{l}\text { Evaluated Nuclear Structure Data File: Recommended nuclear data for 2,916 } \\
\text { nuclides, organized in over 15,000 individual datasets. It serves as a principal } \\
\text { source of data for nuclear structure research, nuclear spectroscopy applications, } \\
\text { MIRD, NuDat, and publications such as Nuclear Data Sheets and Table of } \\
\text { Isotopes. }\end{array}$ \\
\hline XUNDL & www.nndc.bnl.gov/ensdf/ & $\begin{array}{l}\text { Experimental Unevaluated Nuclear Data List: Experimental nuclear structure } \\
\text { and decay data covering more than } 1,100 \text { recent articles. }\end{array}$ \\
\hline MIRD & www.nndc.bnl.gov/mird/ & $\begin{array}{l}\text { Medical Internal Radiation Dose: Recommended nuclear decay data for over } \\
\text { 2,100 radionuclides extracted from ENSDF, processed by the program RadList [1], } \\
\text { and presented in the Medical Internal Radiation Dose format. }\end{array}$ \\
\hline NuDat & www.nndc.bnl.gov/nudat/ & $\begin{array}{l}\text { Nuclear Data: Recommended nuclear structure and decay information for 2,918 } \\
\text { nuclides, about } 136,000 \text { levels, } 197,000 \gamma \text { 's, etc. obtained from ENSDF and the } \\
\text { Nuclear Wallet Cards [2] and thermal neutron data from Neutron Cross Sections } \\
\text { [3]. }\end{array}$ \\
\hline NSR & www.nndc.bnl.gov/nst/ & $\begin{array}{l}\text { Nuclear Science References: Bibliographical nuclear physics information } \\
\text { containing over } 175,000 \text { nuclear science articles, indexed according to content. It } \\
\text { spans almost } 100 \text { years of research, and currently covers } 75 \text { journals with about } \\
4,000 \text { new articles added each year. }\end{array}$ \\
\hline
\end{tabular}

and Java server pages (jsp) or servlets. Combined with new powerful and dedicated Web and database servers, these upgrades have resulted in dramatic improvement in speed of retrieval. Other NNDC Web pages have also been modernized to provide a better and more consistent style (See Figure 1 for our new home page). The improved database services and other resources available from www.nndc.bnl.gov will be described.

\section{- NUCLEAR PHYSICS DATABASES}

The NNDC maintains two bibliographic and six numeric databases covering low and medium energy nuclear physics. ${ }^{4}$ Additional details of the interfaces to our more popular databases follow with an emphasis on new or improved capabilities (New search parameters are underlined).

\section{CSISRS}

Search parameters include target, projectile, product, nuclear reaction, energy range, authors, publication year, and modification date. Improvements include extended and advanced search options for users familiar with EXFOR (Exchange Format) and enhanced plotting capabilities.

\footnotetext{
${ }^{4}$ See Table 1 for brief descriptions of these databases. Most of these are a result of the work of national (Cross Section Evaluation Working Group, CSEWG, and U.S. Nuclear Data Program, USNDP) or international networks (Nuclear Reaction Data Centers Network, NRDC; International Network of Nuclear Structure and Decay Data Evaluators, NSDD); and Working Party on International Nuclear Data Evaluation Cooperation, NRDC).
} 


\section{ENDF}

Search parameters include target, projectile, product, nuclear reaction, energy range, quantity, and library. Improvements include advanced search options for users familiar with the ENDF (Evaluated Nuclear Data File) format and improved plotting capabilities including the ability to overlay CSISRS and ENDF data.

\section{ENSDF/XUNDL}

There is a tighter integration of the ENSDF and XUNDL databases allowing the use of a common interface. Search parameters include nuclide, nuclear reaction, and nuclear decay. Datasets may also be browsed by element or mass.

\section{NuDat}

Search parameters include nuclide or parent, energy levels, decay modes, $J^{\pi}$ 's, $T_{1 / 2}$ 's, $E_{\gamma}$ 's, and $\gamma / \gamma-$ coincidences. Enhancements include a Chart of the Nuclides interface to ground and metastable state properties and options to obtain tables and interactive level schemes of the adopted data contained in ENSDF.

\section{NSR}

Quick retrievals by author or nuclide as well as indexed, text search, and keynumber retrievals, are available. Search parameters for indexed retrievals include nuclide, author, subject, reaction, target, incident or outgoing particle, and topic. A new feature for the Web is the ability to combine previous results using Boolean operations.

\section{OTHER RESOURCES}

We also provide access to many other resources of interest to the nuclear scientist. These include calculational tools, computer codes, data libraries, and publications. $^{5}$

\footnotetext{
${ }^{5}$ See www.nndc.bnl.gov/toolspublications/toolspublications.html for a complete list.
}

\section{Calculational Tools}

\author{
Physics Codes (PhysCo)
}

PhysCo allows for the interactive calculation of $\beta^{ \pm}$ and electron-capture $\log f t$ 's, average $\beta$ energies, etc. or $\gamma$-ray internal conversion coefficients using the programs LOGFT [4] and HSICC [5], respectively. One may also upload a file in the ENSDF format and obtain a new file containing the results of the calculations. See www.nndc.bnl.gov/physco/.

\section{Q-Value Calculator (QCalc)}

QCalc allows the user to calculate decay Q-values or reaction Q-values and threshold energies using the data from the 1995 Update to the Atomic Mass Evaluation [6] or obtain quantities given in this evaluation. See www.nndc.bnl.gov/qcalc/.

A new version of QCalc should be available by the time of this meeting using jsp and the data from the 2003 Atomic Mass Evaluation [7].

\section{Computer Programs}

The ENSDF analysis and utility codes and ENDF utility codes maintained by the NNDC on behalf of the NSDD and CSEWG, respectively, may be downloaded. In addition, nuclear reaction model codes such as EMPIRE and PRECO-2000 are available. See www.nndc.bnl.gov/nndescr/.

\section{Data Libraries}

\section{Atomic Masses}

The Atomic Masses site has been updated to provide the files associated with the 2003 Atomic Mass Evaluation [7]. Archival versions of earlier evaluations are also available. See www.nndc.bnl.gov/masses/.

\section{Point2004}

POINT 2004, A Temperature Dependent ENDF/BVI, Release 8, Cross Section Library [8] was added in April 2004 and consists of data at eight nuclear reactor temperatures between $0^{\circ}$ and $2100^{\circ}$ Kelvin and six astrophysics temperatures between $0.1 \mathrm{eV}$ and $10 \mathrm{keV}$. See www.nndc.bnl.gov/point2004/. 


\section{Publications}

An index to recent issues of the Nuclear Data Sheets, data from the Nuclear Wallet Cards, various manuals, proceedings of national data network meetings, and preprints of publications are available.

\section{Nuclear Wallet Cards}

The Nuclear Wallet Cards site has been significantly expanded. Besides a periodic table interface to the HTML's corresponding to the sixth edition [2], there is also a periodic table interface to the current data in the Wallet Cards module of NuDat, the ability to download a file of the data in PDA-adaptable Palm Pilot format, and a new booklet (Nuclear Wallet Cards for Radioactive Nuclides [9]) and associated file in PDA-adaptable Palm Pilot format. PDF versions of both booklets are also available along with archival versions of previous editions of the Nuclear Wallet Cards. See www.nndc.bnl.gov/wallet/.

\section{Thermal Neutron Capture $\gamma$ 's (CapGam)}

Recently updated, the data on this site are extracted from ENSDF and consist of target and $\gamma$-energy ordered tables for 256 target nuclides up to about $12 \mathrm{MeV}$ in energy. See www.nndc.bnl.gov/capgam/.

\section{ACKNOWLEDGMENTS}

This work was performed under the auspices of U.S.D.O.E., Contract No. DE-AC02-98CH10886.

\section{REFERENCES}

1. Burrows, T. W., The Program RADLST, Brookhaven National Laboratory Report BNL-NCS-52142, February 29, 1988.

2. Tuli, J. K., Nuclear Wallet Cards, January 2000.

3. Mughabghab, S. F., Divadeenam, M., Holden, N. E., Neutron Cross Sections, Vol.1, Neutron Resonance Parameters and Thermal Cross Sections, Part A, $Z=1-60$, Academic Press, New York (1981). Mughabghab, S. F., Neutron Cross Sections, Vol. 1, Neutron Resonance Parameters and Thermal Cross Sections, Part B, Z=61100, Academic Press, New York (1984).

4. Gove, N. B. and Martin, M. J., Nuclear Data Tables A10, 205 (1971).

5. Hager, R. S. and Seltzer, E. C., Nuclear Data Tables A1, 1 (1968).

6. Audi, G., Wapstra, A.H., Nuclear Physics A595, 409 (1995).

7. Wapstra, A.H., Audi, G., and Thibault, C., Nuclear Physics A729, 129 (2003). Audi, G., Wapstra, A.H., and Thibault, C., Nuclear Physics A729, 337 (2003).

8. Cullen, D. E., POINT 2004, A Temperature Dependent ENDF/B-VI, Release 8 Cross Section Library, Lawrence Livermore National Laboratory Report UCRL-TR202284, April 1, 2004.

9. Tuli, J. K., Nuclear Wallet Cards for Radioactive Nuclides, March 2004. 


\section{DISCLAIMER}

This report was prepared as an account of work sponsored by an agency of the United States Government. Neither the United States Government nor any agency thereof, nor any of their employees, nor any of their contractors, subcontractors, or their employees, makes any warranty, express or implied, or assumes any legal liability or responsibility for the accuracy, completeness, or any third party's use or the results of such use of any information, apparatus, product, or process disclosed, or represents that its use would not infringe privately owned rights. Reference herein to any specific commercial product, process, or service by trade name, trademark, manufacturer, or otherwise, does not necessarily constitute or imply its endorsement, recommendation, or favoring by the United States Government or any agency thereof or its contractors or subcontractors. The views and opinions of authors expressed herein do not necessarily state or reflect those of the United States Government or any agency thereof. 